HUTP-99/A039, NUB 3203

\title{
TeV-scale Supersymmetric Standard Model with Higgs as a Slepton
}

\author{
Zurab Kakushadze* \\ Jefferson Laboratory of Physics, Harvard University, Cambridge, MA 02138 \\ and \\ Department of Physics, Northeastern University, Boston, MA 02115
}

(August 2, 1999)

\begin{abstract}
Recently it was pointed out that in the $\mathrm{TeV}$-scale brane world there is a logical possibility where the electroweak Higgs can be identified with a fourth generation slepton. In this paper we address various issues in this four-generation TeV-scale Supersymmetric Standard Model with Higgs as a slepton. In particular we discuss how to achieve proton stability by suppressing the corresponding baryon number violating operators via gauging (a discrete subgroup of) the baryon number $U(1)$ symmetry. Dimension five lepton number violating operators which would result in unacceptably large neutrino masses can be similarly suppressed via gauging a discrete subgroup of the lepton number $U(1)$ symmetry. In fact, the four generation feature allows for a novel higher dimensional mechanism for generating small Majorana neutrino masses. We also discuss how to achieve gauge coupling unification, which can be as precise at one loop as in the MSSM, and point out a possible geometric embedding of the corresponding matter content in the brane world context. Finally, we discuss adequate suppression of flavor changing neutral currents in this model, and also point out a novel possibility for supersymmetry breaking via a non-zero F-term of the fourth generation lepton superfield.
\end{abstract}

Typeset using REVTEX

*E-mail: zurab@string.harvard.edu. Address after September 1, 1999: C.N. Yang Institute for Theoretical Physics, State University of New York, Stony Brook, NY 11794. 


\section{INTRODUCTION}

The electroweak symmetry is believed to be broken via the Higgs mechanism. In the nonsupersymmetric Standard Model one can introduce a complex scalar field that can play the role of the Higgs. In the supersymmetric extensions of the Standard Model, however, adding a single chiral Higgs superfield is not possible due to the corresponding gauge anomaly.

If one does not wish to introduce superfields with exotic quantum numbers, then the anomaly cancellation allows only two possibilities. We can introduce either "vector-like" Higgs pairs or complete generations. The former possibility has been explored in detail, and the supersymmetric extension of the Standard Model with one "vector-like" Higgs pair is known as the Minimal Supersymmetric Standard Model (MSSM). On the other hand, identifying the Higgs as a part of a complete generation is also a logical possibility. This possibility stems from the fact that the $S U(3)_{c} \otimes S U(2)_{w} \otimes U(1)_{Y}$ quantum numbers of the electroweak Higgs are the same as those of an $S U(2)_{w}$ doublet lepton. Thus, one can attempt to identify the electroweak Higgs with the scalar superpartner of the electroweak doublet lepton (that is, the corresponding slepton) in one of the generations.

It is, however, non-trivial to realize this Higgs as slepton scenario. First, within the usual paradigm where the fundamental Planck scale $M_{P} \sim 10^{19} \mathrm{GeV}$ this is impossible due to the well known rules for writing supersymmetric Lagrangians. Thus, in such a scenario the up-quark masses would have to be generated via the couplings of the form $L^{*} Q U$, which are suppressed by the factor $M_{\mathrm{SUSY}}^{2} / M_{P}^{2} \sim 10^{-32}$.

However, the situation is rather different if the fundamental Planck scale $M_{P f}$ is around a $\mathrm{TeV}$ [1]. In particular, now the desired couplings of the slepton Higgs to the Standard Model fermions can be generated via the couplings of the form $X^{*} L^{*} Q U$ in the Kähler potential, where $X$ is the "spurion" superfield whose F-term $\left\langle F_{X}\right\rangle \sim M_{P f}^{2}$ breaks supersymmetry. Note that in this context no additional suppression appears in the corresponding Yukawa couplings. This observation was recently utilized in [2] where the Higgs as a slepton scenario was proposed.

There is, however, another non-trivial constraint arising from the neutrino masses. Thus, we cannot identify the Higgs with the superpartner of a known lepton, which is due to the fact that, via a mixing with neutral gauginos, the corresponding neutrino, which is the superpartner of the neutral Higgs, invariably acquires a see-saw type of mass of order of 10 $\mathrm{GeV}$. For this reason, in [2] it was proposed that there exists the fourth generation, and the Higgs is identified with the corresponding slepton.

In [2] it was shown that such a scenario is not ruled out by the present data, but at the same time can conceivably be tested by LEP-II or the Tevatron. In particular, the fourth generation neutrino picks up a see-saw type of mass, which one requires to be at least 45 $\mathrm{GeV}$ to comply with the Z-decay data. This then implies that at least one out of three neutralinos in this scenario is lighter than $65 \mathrm{GeV}$. In fact, the neutralino production cross sections appear to be testable by the LEP-II data.

Even though the above scenario is not excluded experimentally, it is desirable to address various issues invariably arising in the context of $\mathrm{TeV}$-scale quantum gravity. Thus, the purpose of this paper is to address within the above proposal some of these issues such as proton stability, neutrino masses, gauge coupling unification as well as suppression of flavorchanging neutral currents (FCNCs). These issues were discussed in detail in the context of 
the three-generation TeV-scale Supersymmetric Standard Model (TSSM) proposed in [3]. The gauge coupling unification in the TSSM, which is as precise at one loop as in the MSSM, was discussed in [3] in the context of the higher dimensional scenario proposed in [⿶ㅐㅂ where gauge coupling unification occurs via Kaluza-Klein (KK) thresholds. Higher loop effects were shown to be subleading in this context due to the underlying $\mathcal{N}=2$ supersymmetry at the heavy KK levels in [3: [7. Proton stability and adequate suppression of dimension five lepton number violating operators (which would lead to unacceptably large Majorana masses for neutrinos) was achieved in in the TSSM in [8] by gauging an anomaly free discrete $\mathbf{Z}_{3} \otimes \mathbf{Z}_{3}$ gauge symmetry. Finally, adequate suppression of FCNCs in the TSSM was discussed in [9], in particular, via introducing an anomaly free non-Abelian discrete flavor symmetry $T_{L} \otimes T_{R}$ (here $T$ is the tetrahedral subgroup of $S U(2)$ ) accompanied by an Abelian (continuous or discrete) bulk flavor symmetry.

In this paper we discuss the above issues in the context of the four-generation $\mathrm{TeV}$-scale Supersymmetric Standard Model with Higgs as a fourth-generation slepton. To distinguish it from the TSSM, we will refer to this model as the TSSM4. As we will discuss in the remainder of this paper, proton stability in the TSSM4 can be guaranteed by gauging an anomaly free bulk $U(1)_{\mathcal{B}}$ symmetry related to the baryon number. This, in fact, was originally proposed in 110. However, this $U(1)_{\mathcal{B}}$ symmetry must be broken, and, if it is broken completely, then in certain cases there might be induced $\Delta B=1$ dimension five operators of the form $Q Q Q L / M_{P}$ (here $M_{P}$ is the four dimensional Planck scale), which are well known to be disastrous for proton stability via a one-loop graph involving a chargino exchange [11]. To guarantee proton stability we propose gauging a discrete subgroup of $U(1)_{\mathcal{B}}$ very much along the lines of $[8]$. In fact, it actually suffices to gauge a discrete $\mathbf{Z}_{2}$ subgroup of $U(1)_{\mathcal{B}}$ for this purpose. Other dangerous higher dimensional baryon number violating operators, namely, those with $\Delta B=2$, are not forbidden by this $\mathbf{Z}_{2}$ discrete symmetry. To achieve their suppression one can gauge a $\mathbf{Z}_{4}$ (instead of $\mathbf{Z}_{2}$ ) subgroup of $U(1)_{\mathcal{B}}$. In fact, for this purpose it suffices to have the unbroken $\mathbf{Z}_{2}$ discrete symmetry with the $U(1)_{\mathcal{B}} \rightarrow \mathbf{Z}_{2}$ (or even $\mathbf{Z}_{4} \rightarrow \mathbf{Z}_{2}$ ) breaking taking place on a distant brane.

Suppression of the dangerous dimension five lepton number violating operators in the TSSM4 is achieved by gauging a discrete $\mathbf{Z}_{3}$ subgroup of an anomaly free $U(1)_{\mathcal{L}}$ symmetry related to the lepton number. As we point out in the following, gauging the full $U(1)_{\mathcal{L}}$ symmetry in the TSSM4 context is not possible for the reason that the Higgs is identified with the corresponding fourth generation slepton, and the desired Yukawa couplings would be absent if $U(1)_{\mathcal{L}}$ were gauged in the bulk. Its $\mathbf{Z}_{3}$ subgroup, however, which is completely adequate for the purposes of suppressing dimension five lepton number violating operators, is compatible with the Yukawa couplings of the Higgs with the Standard Model fermions.

The gauge coupling unification in the TSSM4 is based on the same idea as in the TSSM, but a concrete realization is a bit more non-trivial. In particular, we discuss possible geometric embeddings (in terms of orbifold compactifications) of the TSSM4 (with its discrete symmetries) in the brane world context. (For general discussions of embedding the TeVscale quantum gravity scenario in the brane world context, see [10,12, 13].) Finally, we also

\footnotetext{
${ }^{1} \mathrm{TeV}$-scale compactifications were originally discussed in [5] in the context of supersymmetry breaking. Kaluza-Klein threshold corrections to gauge couplings were first studied in [6].
} 
discuss how to suppress FCNCs in the TSSM4 (which is very much along the lines of 99 with some differences due to the presence of the fourth generation) as well as some other issues.

\section{TSSM4}

In this section we briefly review the TSSM4 proposed in [2]. The gauge group of this model is the same as in the MSSM (or the TSSM), that is, $S U(3)_{c} \otimes S U(2)_{w} \otimes U(1)_{Y}$. The light spectrum 2 of the model is $\mathcal{N}=1$ supersymmetric, and along with the vector superfields transforming in the adjoint of $S U(3)_{c} \otimes S U(2)_{w} \otimes U(1)_{Y}$ we also have the following chiral superfields:

$$
\begin{aligned}
& Q_{i}=4 \times(\mathbf{3}, \mathbf{2})(+1 / 3), \quad D_{i}=4 \times(\overline{\mathbf{3}}, \mathbf{1})(+2 / 3), \quad U_{i}=4 \times(\overline{\mathbf{3}}, \mathbf{1})(-4 / 3), \\
& L_{i}=4 \times(\mathbf{1}, \mathbf{2})(-1), \quad E_{i}=4 \times(\mathbf{1}, \mathbf{1})(+2), \quad N_{i}=4 \times(\mathbf{1}, \mathbf{1})(0)
\end{aligned}
$$

Here the $S U(3)_{c} \otimes S U(2)_{w}$ quantum numbers are given in bold font, whereas the $U(1)_{Y}$ hypercharge is given in parentheses. The four generations $(i=1,2,3,4)$ of quarks and leptons are given by $Q_{i}, D_{i}, U_{i}$ respectively $L_{i}, E_{i}, N_{i}$ (the chiral superfields $N_{i}$ correspond to the right-handed neutrinos $\left.{ }^{3}\right)$. The massive KK spectrum will be described in section IV where we discuss the gauge coupling unification in the TSSM4.

In the first approximation we assume that there is no mixing (at least in the lepton sector) between the first three $(i=1,2,3)$ and the fourth generations. In the next section we will discuss how to achieve this using (discrete) gauge symmetries. Then we identify the first three generations with the known quarks and leptons, whereas the heavy fourth generation is new. The fourth generation superfield $L_{4}$ is then identified with the electroweak Higgs superfield.

Next, let $X$ be the "spurion" superfield whose F-term breaks supersymmetry. The downquark masses are generated via the usual couplings in the superpotential which are of the following type:

$$
Q_{i} D_{j} L_{4}, \quad i, j=1,2,3,4
$$

The up-quark masses, however, are generated via the following couplings in the Kähler potential:

$$
Q_{i} U_{j} L_{4}^{*} X^{*} / M_{s}^{2}, \quad i, j=1,2,3,4
$$

\footnotetext{
${ }^{2}$ By the light spectrum we mean the states which are massless before the supersymmetry/electroweak symmetry breaking.

${ }^{3}$ As we will point out in the next section, Majorana masses for neutrinos can be generated in the TSSM4 without introducing bulk right-handed neutrinos but via a novel higher dimensional mechanism.
} 
If the F-term $\left\langle F_{X}\right\rangle \sim M_{s}^{2}$, where the string scale $M_{s}$ is in the TeV ranget, then the up-quark Yukawa couplings are not suppressed.

The charged lepton masses for the first three generations are generated via the usual couplings in the superpotential:

$$
L_{a} E_{b} L_{4}, \quad a, b=1,2,3 .
$$

Note that the analogous couplings for the fourth generation such as $L_{4} E_{4} L_{4}$ are absent due to antisymmetry of the $S U(2)_{w}$ contractions. Thus, the desired Yukawa coupling for the fourth generation charged lepton must come from the Kähler potential. One possible coupling of this type is the following:

$$
(\mathcal{D} X) X^{*} L_{4}\left(\mathcal{D} L_{4}\right) E_{4} / M_{s}^{4}
$$

Once again, this coupling is not suppressed as long as $\left\langle F_{X}\right\rangle \sim M_{s}^{2}$. Generation of neutrino masses will be discussed in the next section.

Finally, let us note that a light Higgs is no longer a prediction of supersymmetry in the present context. Thus, in the TSSM4 the Higgs potential can come from the following terms in the Kähler potential (here $C$ is a model dependent numerical constant)

$$
\left(X X^{*} / M_{s}^{2}\right)\left(L_{4} L_{4}^{*}+C\left(L_{4} L_{4}^{*}\right)^{2} / M_{s}^{2}+\ldots\right)
$$

which can in principle tolerate any mass the Higgs could have in the context of the nonsupersymmetric Standard Model.

\section{PROTON STABILITY AND NEUTRINO MASSES}

In this section we discuss proton stability and generation of small neutrino masses in the TSSM4. In particular, we consider gauging $U(1)_{\mathcal{B}}$ and $U(1)_{\mathcal{L}}$ bulk symmetries (or, more precisely, discrete subgroups thereof) corresponding to the baryon respectively lepton numbers.

\section{A. Proton Stability}

To ensure proton stability within the TSSM4, the simplest possibility is to gauge the baryon number. Note that in the context of three-generation models this is problematic as the corresponding $U(1)$ symmetry would be anomalous. However, with four generations we can have an anomaly free $U(1)$ gauge symmetry corresponding to the baryon number [10]. Indeed, consider the following $U(1)_{\mathcal{B}}$ charge assignments:

\footnotetext{
${ }^{4}$ Here and in the following we assume that the string coupling is of order one, so that the string scale $M_{s}$ and the fundamental Planck scale $M_{P f}$ are of the same order of magnitude. Moreover, by "TeV range" we do not necessarily mean that $M_{s}$ is of order of a few $\mathrm{TeV}$, but can be $\sim 10-100$ $\mathrm{TeV}$. In fact, as was pointed out in [3], which we will re-iterate in the following, this range for the string scale might be preferred from various phenomenological considerations.
} 


$$
\begin{aligned}
& Q_{a}:+1, \quad D_{a}:-1, \quad U_{a}:-1, \quad a=1,2,3, \\
& Q_{4}:-3, \quad D_{4}:+3, \quad U_{4}:+3, \\
& L_{i}: 0, \quad E_{i}: 0, \quad N_{i}: 0, \quad i=1,2,3,4 .
\end{aligned}
$$

Note that the above $U(1)_{\mathcal{B}}$ charge assignment is such that $\mathcal{B}=3 B$ for the first three generations, where $B$ is the usual baryon number. The $\mathcal{B}$ charge assignment for the fourth generation quarks is dictated by the anomaly cancellation requirement. Cancellation of the $U(1)_{\mathcal{B}}$ anomalies can be seen by noting that the above $U(1)_{\mathcal{B}}$ charge assignment can be viewed as descending from a "vector-like" $S U(4)$ "flavor" symmetry with the left handed quarks transforming in $\mathbf{4}$ of $S U(4)$, and the left-handed anti-quarks transforming in $\overline{\mathbf{4}}$.

Note that the $U(1)_{\mathcal{B}}$ symmetry must somehow be broken or else it would lead to a new long-range force contradicting the data. Here we have a few different possibilities. First, we can break $U(1)_{\mathcal{B}}$, which in this case is a bulk gauge symmetry, on a distant brane, so that this breaking is communicated to the brane on which the Standard Model fields are localized via the large extra dimensional bulk fields. The $U(1)_{\mathcal{B}}$ gauge symmetry breaking can then be (almost) maximal on the distant brane, while on our brane it is suppressed by the corresponding factor related to the volume of the large extra dimensions. It is not difficult to see that the baryon number violating operators are suppressed by the appropriate powers of $M_{s} / M_{P}$ (where $M_{P}$ is the four dimensional Planck scale) provided that the separation between the branes is of order of the size of the large extra dimensions. Moreover, the $U(1)_{\mathcal{B}}$ gauge boson picks up a mass of order of or larger than an inverse millimeter leading to a new force which could be observable in the sub-millimeter range [5, 1].

Note, however, that if we break the $U(1)_{\mathcal{B}}$ gauge symmetry completely, then in certain cases there might be induced $\Delta B=1$ dimension five operators of the form $Q Q Q L / M_{P}$ without any additional suppression. Such operators are disastrous for proton stability in the supersymmetric context (which we adapt) via a one-loop graph involving a chargino exchange [11]. Thus, to guarantee proton stability, instead of breaking $U(1)_{\mathcal{B}}$ completely, we can consider breaking it to its discrete subgroup which would forbid such dimension five operators. Alternatively, we may completely bypass $U(1)_{\mathcal{B}}$ and directly consider gauging such a discrete symmetry. In fact, a $\mathbf{Z}_{2}$ subgroup $\widetilde{\mathcal{B}}_{2}$ (in the following we will denote the $\mathbf{Z}_{N}$ subgroup of any $U(1)_{\mathcal{A}}$ gauge symmetry by $\left.\widetilde{\mathcal{A}}_{N}\right)$ suffices for these purposes ${ }^{\text {F }}$. Moreover, if we gauge the $\widetilde{\mathcal{B}}_{2}$ subgroup, then proton is completely stable. Indeed, the higher dimensional operators responsible for proton decay can be schematically written as $Q Q Q L^{k+1}\left(L_{4}^{*}\right)^{k}$, and violate the baryon number by $\Delta B=1$, that is, they violate the $\mathcal{B}$ number by $\Delta \mathcal{B}=3$. If, however, the $\mathcal{B}$ number is conserved modulo 2 , which is the case when we gauge the $\widetilde{\mathcal{B}}_{2}$ discrete subgroup, then these operators are all forbidden, so that proton is completely stable.

Note, however, that $\Delta B=2$ processes would be allowed by the $\widetilde{\mathcal{B}}_{2}$ symmetry. These must also be suppressed as there are experimental bounds on such processes (for instance, from the $N N \rightarrow \pi \pi$ transitions, where $N=p, n)$. The corresponding higher dimensional operators can be completely suppressed if instead of the $\mathbf{Z}_{2}$ subgroup $\widetilde{\mathcal{B}}_{2}$ of $U(1)_{\mathcal{B}}$ we gauge

\footnotetext{
${ }^{5}$ Note that this $\mathbf{Z}_{2}$ discrete symmetry also forbids dimension four baryon number violating operators of the form $U D D$.
} 
its $\mathbf{Z}_{4}$ subgroup $\widetilde{\mathcal{B}}_{4}$. Indeed, $\Delta B=2$ implies $\Delta \mathcal{B}=6$, and since the $\mathcal{B}$ number is conserved modulo 4 in this case, all such processes are forbidden.

Finally, let us point out a "hybrid" possibility which might be of interest in the light of the discussion in section III, where we consider possible geometric embeddings of the TSSM4 in the brane world context. Thus, consider gauging the $\widetilde{\mathcal{B}}_{2}$ discrete symmetry with the $U(1)_{\mathcal{B}} \rightarrow \widetilde{\mathcal{B}}_{2}$ breaking taking place on a distant brane 9 . Then proton would be completely stable but the $\Delta B=2$ baryon number violating processes would be allowed albeit adequately suppressed by the appropriate powers of $M_{s} / M_{P}$.

The above "hybrid" possibility might be interesting in the context of baryogenesis. It seems non-trivial to have successful baryogenesis in the context of $\mathrm{TeV}$-scale brane world for the following reason. On the one hand, baryon number violating processes must be adequately suppressed by various (discrete) gauge symmetries, and the allowed baryon number operators then have rather high dimensions. On the other hand, the reheat temperature cannot be too high in the context of $\mathrm{TeV}$-scale brane world or else one generically runs into various cosmological problems (for instance, with the bulk gravitons) [1]. Here we would like to point out the following possibility. In the above "hybrid" scenario, where $\widetilde{\mathcal{B}}_{2}$ is unbroken but $U(1)_{\mathcal{B}}$ (or $\left.\widetilde{\mathcal{B}}_{4}\right)$ is broken down to $\widetilde{\mathcal{B}}_{2}$ on a distant brane, the dimension five operators $Q Q Q L$ are forbidden, but the operators of the form $Q Q Q Q Q Q L_{4} L_{4}$ (after the electroweak breaking) give rise to effective six-fermion operators (which violate the baryon number by $\Delta B=2$ ) suppressed by $\eta M_{\mathrm{EW}}^{2} / M_{s}^{7}$. Here the additional suppression factor $\eta$ (which is given by the appropriate powers of $M_{s} / M_{P}$ ) is due to the fact that the above breaking occurs on a distant brane. However, in the early universe the branes can be much closer to each other. In fact, even though the sizes of the large extra dimensions can already be fixed at their zero temperature values, the brane stabilization need not have taken place yet as the latter may be due to dynamics different from that responsible for radius stabilization. (For recent discussions on brane stabilization in the brane world context, see, e.g., 14,15.).) Thus, when the branes are at distances of order $1 / M_{s}$ from each other, the factor $\eta \sim 1$, so that there is no extra suppression in the above effective six-fermion operators. We should point out that it is still not completely clear whether one can have successful baryogenesis in this context, and it would be interesting to understand this issue in more detail. (For recent discussions on baryogenesis in the context of $\mathrm{TeV}$-scale brane world, see, e.g., 116 18.)

\section{B. Neutrino Masses}

To generate small (Dirac) neutrino masses in the TSSM4, we can assume that the righthanded neutrinos $N_{i}$ are bulk fields, and then the neutrino masses are generated via the higher dimensional mechanism of [19]. However, as was pointed out in [8], for this mechanism to work one must adequately suppress dangerous (effective) dimension five lepton number violating operators which in the TSSM4 context have the following form: $L_{a} L_{b} L_{4}^{*} L_{4}^{*}, a, b=$ 1, 2,3. Here we take fermionic components for $L_{a}$ and $L_{b}$, and bosonic components for both

\footnotetext{
${ }^{6}$ Alternatively, we could imagine that we start with the $\widetilde{\mathcal{B}}_{4}$ symmetry broken on a distant brane to its $\mathbf{Z}_{2}$ subgroup $\widetilde{\mathcal{B}}_{2}$.
} 
$L_{4}^{*}$ 's. If these operators are suppressed only by $1 / M_{s}$, then upon the electroweak symmetry breaking (that is, once the $L_{4} \mathrm{VEV}$ is non-zero) such operators would generate unacceptably large Majorana neutrino masses of order $M_{\mathrm{EW}}^{2} / M_{s}$ for the first three generation neutrinos. On the other hand, the analogous operator of the form $L_{4} L_{4} L_{4}^{*} L_{4}^{*}$ is welcome. In fact, the "see-saw" type of mass for the fourth generation neutrino amounts to generating precisely such an operator via the mixing of the fourth generation neutrino with the neutral gauginos (whose linear combination in this case plays the role of the corresponding "right-handed" neutrino).

To suppress the operators of the form $L_{a} L_{b} L_{4}^{*} L_{4}^{*}$, the simplest possibility is to gauge the lepton number. Note that, just as in the case of the baryon number, in the context of the three-generation models this is problematic as the corresponding $U(1)$ symmetry would be anomalous. However, with four generations we can have an anomaly free $U(1)$ gauge symmetry corresponding to the lepton number. Indeed, consider the following $U(1)_{\mathcal{L}}$ charge assignments:

$$
\begin{aligned}
& Q_{i}: 0, \quad D_{i}: 0, \quad U_{i}: 0, \quad i=1,2,3,4, \\
& L_{a}:+1, \quad E_{a}:-1, \quad N_{a}:-1, \quad a=1,2,3, \\
& L_{4}:-3, \quad E_{4}:+3, \quad N_{4}:+3 .
\end{aligned}
$$

Note that the above $U(1)_{\mathcal{L}}$ charge assignment is such that $\mathcal{L}=L$ for the first three generations, where $L$ is the usual lepton number. The $\mathcal{L}$ charge assignment for the fourth generation leptons is dictated by the anomaly cancellation requirement. Cancellation of the $U(1)_{\mathcal{L}}$ anomalies is completely analogous to that for $U(1)_{\mathcal{B}}$.

Note that the $U(1)_{\mathcal{L}}$ gauge symmetry forbids the operators of the form $L_{a} L_{b} L_{4}^{*} L_{4}^{*}$ (but allows the operator $\left.L_{4} L_{4} L_{4}^{*} L_{4}^{*}\right)$. Thus, gauging the $U(1)_{\mathcal{L}}$ symmetry would suffice for the purposes of suppressing dangerous dimension five lepton number violating operators. Note, however, that the $U(1)_{\mathcal{L}}$ symmetry is unnecessarily strong for these purposes alone. Moreover, it actually forbids the desirable (effective) Yukawa couplings of the form $Q D L_{4}, Q U L_{4}^{*}$, $L E L_{4}$ as well as $L N L_{4}^{*}$ as $L_{4}$ carries a non-zero $U(1)_{\mathcal{L}}$ charge. To remedy this, instead of gauging the full $U(1)_{\mathcal{L}}$ symmetry, very much in the spirit of [8] we propose to gauge its $\mathbf{Z}_{3}$ subgroup $\widetilde{\mathcal{L}}_{3}$. The $\widetilde{\mathcal{L}}_{3}$ charge assignments are given by:

$$
\begin{aligned}
& Q_{i}: 0, \quad D_{i}: 0, \quad U_{i}: 0, \quad i=1,2,3,4, \\
& L_{a}:+1, \quad E_{a}:-1, \quad N_{a}:-1, \quad a=1,2,3, \\
& L_{4}: 0, \quad E_{4}: 0, \quad N_{4}: 0 .
\end{aligned}
$$

Note that the $\widetilde{\mathcal{L}}_{3}$ charge is conserved modulo 3 . Thus, the operators $L_{a} L_{b} L_{4}^{*} L_{4}^{*}$ are still forbidden, while the (effective) Yukawa couplings $Q D L_{4}, Q U L_{4}^{*}, L E L_{4}$ as well as $L N L_{4}^{*}$ are now allowed.

Gauging the $\widetilde{\mathcal{L}}_{3}$ discrete symmetry allows for an interesting novel higher dimensional mechanism for generating small Majorana masses without introducing bulk right-handed neutrinos. (A similar mechanism was pointed out in 8$]$ in the TSSM context.) Thus, note that the mixing between the first three and the fourth generation leptons is absent if the $\widetilde{\mathcal{L}}_{3}$ discrete symmetry is exact. However, imagine that this symmetry is broken on a distant brane. Then generically there is going to be induced non-zero mixing between the first three and the fourth generation leptons, but this mixing is suppressed by a factor of $M_{s} / M_{*}$, where 
$M_{*}$ (which is roughly of order $M_{P}$, but can actually be a few orders of magnitude smaller) is related to the volume of the large extra dimensions, and with the appropriate separation between the branes can be of the correct order of magnitude so that the induced Majorana masses for the first three generation neutrinos are in the desirable range. (Recall that the fourth generation neutrino has a mass between 45 and $65 \mathrm{GeV}$.) Note that this mechanism for generating small Majorana masses does not require bulk right-handed neutrinos. The role of the fourth generation "right-handed" neutrino is played by a linear combination of neutral gauginos, and the first three generations acquire Majorana masses via the mixing with the fourth generation neutrino. Nonetheless, the presence of the states $N_{i}$ in this scenario is still required by anomaly cancellation, but what is different now is that these states need not be bulk fields. Thus, we can assume that they do not propagate in the large extra dimensions. However, if they are localized on the same brane as the fields $L_{i}$, then we would generically have unacceptably large Dirac neutrino masses coming from the allowed couplings $L N L_{4}^{*}$. This can be remedied by assuming that the states $N_{i}$ are localized on a distant brane (or, alternatively, they come from different fixed points in the context of orbifold compactifications] - see the next section), in which case the above couplings are at least exponentially suppressed.

\section{GAUGE COUPLING UNIFICATION}

In this section we discuss the gauge coupling unification in TSSM4. Since the unification scale, which we identify with the string scale, is now in the $\mathrm{TeV}$ range, the unification mechanism must be very different from that in the MSSM. In [4] such a unification mechanism was proposed. Thus, let the Standard Model fields be localized on D $p$-branes (where $p=4,5)$ with $p-3$ extra directions compactified on a space with linear "size(s)" of order $R$. Let us assume that $R \gg 1 / M_{s}$. Then between the Kaluza-Klein (KK) threshold $1 / R$ and the cut-off $M_{s}$ we have $\sim\left(R M_{s}\right)^{p-3}$ heavy KK modes carrying the corresponding gauge quantum numbers. These states contribute into the gauge coupling running above the scale $1 / R$, and if we are interested in the contributions of these states (that is the corresponding thresholds) into the low energy (that is, at scales $\mu \ll 1 / R \ll M_{s}$ ) gauge coupling renormalization at one loop, they are proportional to the number of these states $N \sim\left(R M_{s}\right)^{p-3} \gg 1$. Thus, one can hope that there might be a possibility of an accelerated unification at $M_{s}$ in the $\mathrm{TeV}$ range.

As was explained in detail in [3.7], this mechanism would have no predictive power unless we adapt the supersymmetric context where the zero modes of the compactification (corresponding, in particular, to the Standard Model fields) are $\mathcal{N}=1$ supersymmetric,

\footnotetext{
${ }^{7}$ Thus, for instance, if we assume that our brane is sitting at an orbifold fixed point while the fields $N_{i}$ come from a different fixed point, then the couplings $L N L_{4}^{*}$ would be suppressed by an exponential factor $\sim \exp \left(-c\left(R M_{s}\right)^{2}\right)$, where $R$ is (roughly) the linear "size" of dimensions along which the orbifold fixed points are separated (and these dimensions are assumed to be large enough so that this exponential factor is adequately suppressed), while $c$ is a model dependent numerical constant of order 1 .
} 
while the heavy $\mathrm{KK}$ modes have $\mathcal{N}=2$ supersymmetry. The reason is that higher loop effects would otherwise be as large as the one-loop threshold contribution as we have a large number $N$ of heavy KK modes circulating in loops. The underlying $\mathcal{N}=2$ supersymmetry at the heavy KK modes guarantees, as was shown in [3, 7], that the higher loop effects are subleading compared with the leading one-loop threshold contribution. This is due to the well known non-renormalization property in $\mathcal{N}=2$ gauge theories. Note, however, that, as was stressed in [3.7], higher loop cancellations due to the underlying supersymmetry persist after supersymmetry breaking only if $M_{\text {SUSY }} \ll 1 / R$. This is one of the key reasons why we assume that $M_{s}$ is at least $10-100 \mathrm{TeV}$.

We will discuss possible geometric embeddings (via orbifolds) of such compactifications in the next subsection. Here let us just assume that the zero modes are $\mathcal{N}=1$ supersymmetric with the one-loop $\beta$-function coefficients $b_{r}, r=1,2,3$ (here $r$ labels the corresponding subgroup of $\left.S U(3)_{c} \otimes S U(2)_{w} \otimes U(1)_{Y}\right)$, while the heavy KK levels are populated by $\mathcal{N}=2$ supermultiplets with the one-loop $\beta$-function coefficients $\widetilde{b}_{r}$. It is then not difficult to show that the unification of gauge couplings in such a model is as precise as in the MSSM (at one loop) if and only if the following constraint is satisfied:

$$
\nu_{r s} \equiv \nu \text { is independent of } r, s, \text { where } \nu_{r s} \equiv \frac{\widetilde{b}_{r}-\widetilde{b}_{s}}{b_{r}^{*}-b_{s}^{*}} \text { for } r \neq s \text {. }
$$

Here $b_{r}^{*}$ are the one-loop $\beta$-function coefficients in the MSSM: $b_{1}^{*}=33 / 5, b_{2}^{*}=1, b_{3}^{*}=-3$ (we are using the standard normalization for the $U(1)_{Y}$ gauge coupling $\left.\alpha_{1}=(5 / 3) \alpha_{Y}\right)$.

In the following we assume that at the heavy $\mathrm{KK}$ levels we have $\mathcal{N}=2$ vector multiplets in the adjoint of the gauge group, and the charged $\mathcal{N}=2$ hypermultiplets can only have the gauge quantum numbers of $Q, D, U, L, E$ (that is, we have no "exotic" matter). Moreover, we will not distinguish between the $Q, D, U, L, E$ and their conjugate quantum numbers as the difference is immaterial as far as the one-loop gauge coupling running is concerned. We will denote the number of the corresponding charged hypermultiplets (per heavy KK mass level) by $n_{Q}, n_{D}, n_{U}, n_{L}, n_{E}$.

The one-loop $\beta$-function coefficients $\widetilde{b}_{r}$ are given by:

$$
\begin{aligned}
& \widetilde{b}_{1}=\frac{1}{5}\left(n_{Q}+2 n_{D}+8 n_{U}+3 n_{L}+6 n_{E}\right), \\
& \widetilde{b}_{2}=-4+3 n_{Q}+n_{L}, \\
& \widetilde{b}_{3}=-6+2 n_{Q}+n_{D}+n_{U} .
\end{aligned}
$$

The constraint (6) can then be rewritten as

$$
\begin{aligned}
& n_{Q}+n_{L}-n_{D}-n_{U}=4 \nu-2, \\
& n_{U}+n_{E}-2 n_{Q}=6 \nu-4 .
\end{aligned}
$$

It is straightforward to analyze this system of algebraic equations, but, instead of being most general here, for illustrative purposes let us consider possible solutions with $\nu=1$. One such solution is $n_{Q}=n_{D}=n_{U}=n_{f}, n_{L}=n_{E}=n_{f}+2$. Note that this precisely corresponds to the TSSM matter content of [3] with $n_{f}$ generations plus $H_{ \pm}$and $F_{ \pm}$hypermultiplets (see [3] for details) propagating in the $p-3$ dimensional bulk (where the gauge bosons live). 
Here we are interested in a four-generation model (that is, the TSSM4), where we do not wish to add any extra "vector-like" states (such as $H_{ \pm}$of the MSSM, or $F_{ \pm}$of the TSSM). One interesting (for the reason we will explain in a moment) solution of this type is the following. Let $n_{Q}=n_{D}=n_{E}=0, n_{U}=2$, and $n_{L}=4$. Then we can imagine the following setup. We have four generations of quarks and leptons only. The states $Q_{i}, D_{i}, E_{i}$, $i=1,2,3,4$, are localized at fixed points (see the next subsection), so that they do not propagate in the $p-3$ dimensional bulk. The same is the case for the states $U_{1}, U_{2}$. The states $U_{3}, U_{4}$ as well as $L_{i}, i=1,2,3,4$, however, are not localized at fixed points, but propagate in the $p-3$ dimensional bulk. Then the unification in the TSSM4 within this setup is as precise at one loop as in the MSSM and the TSSM.

\section{A. Brane World Embedding}

As we mentioned above, the massless modes in the TSSM4 are $\mathcal{N}=1$ supersymmetric, whereas the massive KK modes have $\mathcal{N}=2$ supersymmetry. Such spectra arise in orbifold compactifications in the brane world context, more concretely, in compactifications on generalized Voisin-Borcea orbifolds. Thus, consider Type I/Type I' string theory compactified on an elliptically fibered Calabi-Yau three-fold (with $S U(3)$ holonomy) of the following form: $\mathcal{M}_{3}=\left(T^{2} \otimes \mathrm{K} 3\right) / \mathbf{Z}_{M}$, where the generator $g$ of $\mathbf{Z}_{M}$ (here $M$ can only take values $M=2,3,4,6$ as the action of $g$ on $T^{2}$ must be crystallographic) acts as a $2 \pi / M$ rotation $g z_{1}=\omega z_{1}$ on $T^{2}\left(z_{1}\right.$ is the complex coordinate parametrizing $T^{2}$, and $\left.\omega \equiv \exp (2 \pi i / M)\right)$, and as $g \Omega_{2}=\omega^{-1} \Omega_{2}$ on K3 ( $\Omega_{2}$ is the holomorphic 2-form on K3). Next, consider D5-branes wrapping the fiber $T^{2}$. Then the zero modes of the gauge theory living in the world-volume of the D5-branes are $\mathcal{N}=1$ supersymmetric, while the heavy KK modes are $\mathcal{N}=2$ supersymmetric.

In the following we would like to discuss possible brane world embeddings of the TSSM4 in the context of such orbifold compactifications. In particular, solutions to the constraint (6) imply that some of the matter fields should be localized at fixed points while others propagate in the entire world-volume of D5-branes. It is therefore necessary to check whether any such solution is at least in principle compatible with the geometric embedding via orbifolds?. Here we should stress that the following discussion still does not guarantee that the corresponding model exists as due to the lack of necessary model building technology it is not clear at present whether there exists the appropriate choice of the gauge bundle which would give the desired gauge group and spectrum?. However, as we discuss in the following, the purely

\footnotetext{
${ }^{8}$ This was checked for the TSSM in [3,8]. Subsequently other straightforward variations of the TSSM were discussed in the literature (see, e.g., [20]), where some fields are supposed to be localized at fixed points while others propagate in the bulk. However, it is not enough to simply find a solution to the constraint (6), which is straightforward, but one must also check whether such a solution is at least conceivably embeddable in the brane world context.

${ }^{9}$ Here we would like to point out that, if such an embedding exists, the string coupling is expected to be of order 1 as otherwise it is difficult to imagine how the dilaton could be stabilized [13]. The
} 
geometric part of this issue can be studied by considering possible orbifold compactifications with the corresponding discrete gauge symmetries.

Our discussion in this subsection will be brief as most of the ingredients we are going to use here were discussed in detail in [3]. Thus, we wish to obtain a model where the fields $Q_{i}, D_{i}, E_{i}, U_{1,2}(i=1,2,3,4)$ are localized at fixed points, while the fields $L_{i}, U_{3,4}$ propagate in the bulk (see above). As was pointed out in [3], the fields localized at the orbifold fixed points arise in the Type I/Type I' compactifications in the context of non-perturbative orientifolds examples of which were recently constructed in [21] (for recent progress in perturbative orientifolds, see, e.g., [22]). In particular, these states arise in the twisted sectors, and, therefore, carry the corresponding discrete quantum numbers. Thus, we will first gauge an anomaly free discrete symmetry under which the states $Q_{i}, D_{i}, U_{i}, L_{i}, E_{i}, N_{i}$ have certain discrete gauge charge assignments, and then we will identify this discrete symmetry with that of an orbifold compactification. This will suggest a possible brane world embedding of the TSSM4 with the above matter content.

Our strategy for gauging the desired discrete symmetry will be to first gauge an anomalyfree continuous Abelian symmetry, and then to restrict to its discrete subgroup. Thus, consider the $U(1)_{\mathcal{Z}}$ symmetry, where $\mathcal{Z} \equiv 3(Y-\mathcal{R})$, and the $\mathcal{Z}$ action is the same on all four generations (that is, the $U(1)_{\mathcal{Z}}$ symmetry is generation blind). Here $Y$ is the hypercharge, while the $\mathcal{R}$ charge assignment (which is generation blind) is given by:

$$
\begin{array}{lll}
\mathcal{R}_{Q}=0, & \mathcal{R}_{D}=+1, & \mathcal{R}_{U}=-1 \\
\mathcal{R}_{L}=0, & \mathcal{R}_{E}=+1, & \mathcal{R}_{N}=-1
\end{array}
$$

Note that the $U(1)_{\mathcal{R}}$ symmetry is anomaly free and compatible with the $U_{\mathcal{B}}$ and $U_{\mathcal{L}}$ symmetries. This implies that so is $U(1)_{\mathcal{Z}}$. In fact, the $\mathcal{Z}$ charge assignment is such that $\mathcal{Z}$ acts as $3(B-L)$ on the first three generations.

In the following we will be interested in gauging not $U(1)_{\mathcal{Z}}$ but rather $U(1)_{\mathcal{Z}^{\prime}}$, where $\mathcal{Z}^{\prime} \equiv \mathcal{Z}-\mathcal{R}$. The $U(1)_{\mathcal{Z}^{\prime}}$ charge assignment is given by:

$$
\begin{array}{lll}
\mathcal{Z}_{Q}^{\prime}=+1, & \mathcal{Z}_{D}^{\prime}=-2, & \mathcal{Z}_{U}^{\prime}=0, \\
\mathcal{Z}_{L}^{\prime}=-3, & \mathcal{Z}_{E}^{\prime}=+2, & \mathcal{Z}_{N}^{\prime}=+4 .
\end{array}
$$

Let $\widetilde{\mathcal{Z}}_{3}^{\prime}$ be a $\mathbf{Z}_{3}$ subgroup of $U(1)_{\mathcal{Z}^{\prime}}$, and let $\theta$ be the generator of $\widetilde{\mathcal{Z}}_{3}^{\prime}$. The $\widetilde{\mathcal{Z}}_{3}^{\prime}$ charge assignment is given by (the $\widetilde{\mathcal{Z}}_{3}^{\prime}$ charge is conserved modulo 3 ):

$$
\begin{aligned}
& Q_{i}:+1, \quad D:+1, \quad U_{i}: 0, \\
& L_{i}: 0, \quad E_{i}:-1, \quad N_{i}:+1 .
\end{aligned}
$$

weakness of the Standard Model gauge couplings then requires that the volume of the compact dimensions inside of the D5-branes on which the Standard Model gauge fields are localized be somewhat larger than 1 (in the string units) as is the case in the context of unification via KaluzaKlein thresholds. Note that even though the underlying string theory is in the non-perturbative regime, the corresponding effective field theory is weakly coupled, so that various perturbative considerations such as gauge coupling unification are valid. In fact, in the present context this issue was discussed in detail in [3:7], where the importance of the underlying $\mathcal{N}=2$ supersymmetry at the heavy Kaluza-Klein levels was stressed. 
Here $i=1,2,3,4$, and $\widetilde{\mathcal{Z}}_{3}^{\prime}$ is a generation blind symmetry.

Next, consider the $U(1)_{\mathcal{R}^{\prime}}$ symmetry, where $\mathcal{R}^{\prime}$ acts as $\mathcal{R}$ on the first two generations, while the other two generations have zero $\mathcal{R}^{\prime}$ charges. This symmetry is anomaly free and compatible with all the other symmetries we have considered so far. In the following we will need a $\mathbf{Z}_{2}$ subgroup $\widetilde{\mathcal{R}_{2}^{\prime}}$ of $U(1)_{\mathcal{R}^{\prime}}$, whose generator we will denote by $R$.

The discrete symmetry we are interested in here is the $\mathbf{Z}_{6}$ symmetry $\widetilde{\mathcal{Z}}_{3}^{\prime} \otimes \widetilde{\mathcal{R}}_{2}^{\prime}$. In particular, we will identify the generator $\theta R$ of this group with the generator $g$ of the orbifold group $Z_{M}$ with $M=6$ in the above discussion. Then the states $L_{i}, U_{3,4}$ come from the untwisted sector, the states $Q_{i}, D_{3,4}, N_{3,4}$ come from the $\theta$-twisted (that is, $g^{-2}$-twisted) sector, the states $E_{3,4}$ come from the $\theta^{-1}$-twisted (that is, $g^{2}$-twisted) sector, the states $D_{1,2}, U_{1,2}, N_{1,2}$ come from the $\theta R$-twisted (that is, $g$-twisted) sector, and the states $E_{1,2}$ come from the $\theta^{-1} R$-twisted (that is, $g^{-1}$-twisted) sector. The $R$-twisted (that is, $g^{3}$-twisted sector) does not contain any states. With the above identifications, it is conceivable that the above model may be obtainable as a Type I/Type I' compactification on a generalized VoisinBorcea orbifold of the form $\left(T^{2} \otimes \mathrm{K} 3\right) / \mathbf{Z}_{6}$. Whether the number of the corresponding fields in each sector (as well as their gauge quantum numbers) can come out right is a model building question which depends on an appropriate choice of the gauge bundle (whose existence is a non-trivial issue) as discussed in detail in [3].

Before we end this section, let us point out that the $\widetilde{\mathcal{L}}_{3}$ and $\widetilde{\mathcal{B}}_{2}$ discrete gauge symmetries may also be embeddable in the above context if we take K3 to be an orbifold K3 with the orbifold group being $\mathbf{Z}_{6}^{\prime} \approx \widetilde{\mathcal{L}}_{3} \otimes \widetilde{\mathcal{B}}_{2}$, where the generator $g^{\prime}$ of $\mathbf{Z}_{6}^{\prime}$ acts trivially on the fiber $T^{2}$. Note that we could not embed $\widetilde{\mathcal{B}}_{4}$ instead of $\widetilde{\mathcal{B}}_{2}$ in this context as the total orbifold group in this case could not act crystallographically. This is the reason why we considered a "hybrid" possibility for suppressing baryon number violation in subsection III.A.

Also note that the above $\mathbf{Z}_{6}$ discrete symmetry must actually be broken or else the desirable Yukawa couplings of the Standard Model fermions to the Higgs would be forbidden. This implies that we are actually talking about (at least partially) blown-up orbifolds. In fact, if the breaking of $\mathbf{Z}_{6}$ can be viewed as being due to some fields charged under $\mathbf{Z}_{6}$ acquiring non-zero VEVs in an asymmetric fashion (that is, the VEV of the field with the $+1 \mathbf{Z}_{6}$ charge is different from that of the field with the $-1 \mathbf{Z}_{6}$ charge, which is conceivable in the context of almost completely broken supersymmetry), then this could be used to explain the vertical hierarchy in the quark and lepton sectors.

Finally, we note that if we consider the $\mathbf{Z}_{6} \otimes \mathbf{Z}_{6}^{\prime}$ orbifold, then the right-handed neutrinos can be seen to be localized at " $(3+1)$-dimensional" fixed points, that is, they do not propagate in large extra dimensions in this case. This is one of the reasons why the novel mechanism for generating small Majorana neutrino masses discussed in subsection III.B might be appealing in this context.

\section{OTHER ISSUES}

In this section we would like to briefly discuss some other issues in the TSSM4 such as adequate suppression of FCNCs, and also a novel possibility for supersymmetry breaking.

Suppressing (effective) four-fermion operators potentially inducing unacceptably large FCNCs in the TSSM was discussed in detail in [9]. There it was shown that a non-Abelian discrete flavor group $T_{L} \otimes T_{R}$ (where $T$ is the tetrahedral subgroup of $S U(2)$ ) accompanied 
by a vector-like $U(1)_{V}$ flavor symmetry (or its appropriate discrete subgroup) can adequately suppress all such operators. Here the $T_{L} \otimes T_{R}$ discrete flavor symmetry acts only on the first two generation quarks and leptons, whereas the $U(1)_{V}$ flavor symmetry acts only on the third generation quarks and leptons (in the flavor basis). The former suppresses fourfermion operators involving only the first two generation states, while the latter guarantees that four-fermion operators also involving the third generation states are suppressed as well.

The above mechanism for suppressing FCNCs can also be applied to the TSSM4. The only difference is that we now have the fourth generation, and we must also worry about possible FCNCs coming from the mixing between the first three and the fourth generations. Note, however, that this mixing is guaranteed to be small in the quark sector if the $U(1)_{\mathcal{B}} \rightarrow$ $\widetilde{\mathcal{B}}_{2}$ breaking occurs on a distant brane as in the "hybrid" scenario discussed in subsection III.A. As to the lepton sector, in the limit of unbroken $\widetilde{\mathcal{L}}_{3}$ discrete gauge symmetry the mixing between the first three and the fourth generation leptons is absent, and, since this discrete gauge symmetry is broken on a distant brane, the resulting FCNCs are completely adequately suppressed.

Finally, we would like to point out that in the TSSM4 there a priori exists a novel possibility for supersymmetry breaking. In particular, imagine that not only the lowest (that is, scalar) component of the chiral superfield $L_{4}$ has a non-zero VEV, but also the highest component (that is, the F-term) develops a non-zero VEV. Then the F-term $F_{L_{4}}$ could be responsible for supersymmetry breaking. In fact, in this case we might no longer need a "spurion" superfield $X$ introduced in section II. Thus, the up-quark masses could be generated from the following dimension five operators in the Kähler potential: $Q U L_{4}^{*} / M_{s}$. Indeed, if $\left\langle F_{L_{4}}\right\rangle \sim M_{s}^{2}$, then we have effective Yukawa couplings in the up-quark sector. Similar consideration apply, for instance, to the Yukawa couplings for the fourth generation charged leptons. It would be interesting to investigate this new possibility for supersymmetry breaking in the TSSM4 in more detail.

\section{ACKNOWLEDGMENTS}

I would like to thank Gregory Gabadadze and Aaron Grant for discussions. This work was supported in part by the grant NSF PHY-96-02074, and the DOE 1994 OJI award. I would also like to thank Albert and Ribena Yu for financial support. 


\section{REFERENCES}

[1] N. Arkani-Hamed, S. Dimopoulos and G. Dvali, Phys. Lett. B429 (1998) 263; Phys. Rev. D59 (1999) 0860004.

[2] A.K. Grant and Z. Kakushadze, hep-ph/9906556.

[3] Z. Kakushadze, Nucl. Phys. B548 (1999) 205.

[4] K.R. Dienes, E. Dudas and T. Gherghetta, Phys. Lett. B436 (1998) 55; Nucl. Phys. B537 (1999) 47; hep-ph/9807522.

[5] I. Antoniadis, Phys. Lett. B246 (1990) 317.

[6] T.R. Taylor and G. Veneziano, Phys. Lett. B212 (1988) 147.

[7] Z. Kakushadze and T.R. Taylor, hep-th/9905137.

[8] Z. Kakushadze, Nucl. Phys. B552 (1999) 3.

[9] Z. Kakushadze, Nucl. Phys. B551 (1999) 549.

[10] I. Antoniadis, N. Arkani-Hamed, S. Dimopoulos and G. Dvali, Phys. Lett. B436 (1998) 257.

[11] S. Weinberg, Phys. Rev. D26 (1982) 287;

N. Sakai and T. Yanagida, Nucl. Phys. B197 (1982) 533.

[12] G. Shiu and S.-H.H. Tye, Phys. Rev. D58 (1998) 106007.

[13] Z. Kakushadze and S.-H.H. Tye, Nucl. Phys. B548 (1999) 180.

[14] G. Dvali, hep-ph/9905204.

[15] Z. Kakushadze, hep-th/9906222.

[16] K. Benakli and S. Davidson, Phys. Rev. D60 (1999) 025004.

[17] N. Arkani-Hamed, S. Dimopoulos, N. Kaloper and J. March-Russell, hep-ph/9903224.

[18] G. Dvali and G. Gabadadze, hep-ph/9904221.

[19] N. Arkani-Named, S. Dimopoulos, G. Dvali and J. March-Russell, talk presented by S. Dimopoulos at SUSY98, hep-ph/9811448.

[20] C.D. Carone, Phys. Lett. B454 (1999) 70;

A. Delgado and M. Quirós, hep-ph/9903400;

P.H. Frampton and A. Rašin, hep-ph/9903479.

[21] Z. Kakushadze, Phys. Lett. B455 (1999) 120; hep-th/9904211; hep-th/9905033.

[22] M. Berkooz and R.G. Leigh, Nucl. Phys. B483 (1997) 187;

C. Angelantonj, M. Bianchi, G. Pradisi, A. Sagnotti and Ya.S. Stanev, Phys. Lett. B385 (1996) 96;

Z. Kakushadze, Nucl. Phys. B512 (1998) 221; Nucl. Phys. B529 (1998) 157; Phys. Lett. B434 (1998) 269; Nucl. Phys. B535 (1998) 311; Phys. Rev. D58 (1998) 101901;

Z. Kakushadze and G. Shiu, Phys. Rev. D56 (1997) 3686; Nucl. Phys. B520 (1998) 75;

G. Zwart, Nucl. Phys. B526 (1998) 378;

G. Aldazabal, A. Font, L.E. Ibáñez and G. Violero, Nucl. Phys. B536 (1998) 29;

Z. Kakushadze, G. Shiu and S.-H.H. Tye, Nucl. Phys. B533 (1998) 25;

J. Lykken, E. Poppitz and S.P. Trivedi, Nucl. Phys. B543 (1999) 105;

R. Blumenhagen and A. Wisskirchen, Phys. Lett. B438 (1998) 52;

Z. Kakushadze and S.-H.H. Tye, Phys. Rev. D58 (1998) 126001. 